\title{
Postnatal Evaluation of Congenital Chest Pathologies Using a Low-Dose Computed Tomography (CT) Protocol - a Pictorial Review
}

Authors' Contribution:
A Study Design
B Data Collection
C Statistical Analysis
D Data Interpretation
E Manuscript Preparation
F Literature Search
G Funds Collection

Authors' Contribution:

A Study Design

B Data Collection

C Statistical Analysis

Data Interpretation

F Literature Search

G Funds Collection

\author{
Danuta Roik ${ }^{1 \mathrm{ABCD} F}$, Marzena Barczuk ${ }^{1 \mathrm{BCE}}$, Zofia Burzyńska $^{1 \text { BCE }}$, Agnieszka Biejat ${ }^{1 \mathrm{BC}}$, \\ Maria Żerańska ${ }^{2 \mathrm{~B}}$, Magdalena Mierzewska-Schmidt ${ }^{3 \mathrm{~B}}$, Tomasz Floriańczyk ${ }^{4 \mathrm{~B}}$, \\ Michał Brzewski ${ }^{1 \mathrm{ABD}}$ \\ ${ }^{1}$ Department of Pediatric Radiology, Medical University of Warsaw, Warsaw, Poland \\ 2 Department of Pediatric Surgery, Medical University of Warsaw, Warsaw, Poland \\ ${ }^{3}$ Department of Pediatric Anesthesiology and Intensive Therapy, Medical University of Warsaw, Warsaw, Poland \\ ${ }^{4}$ Department of Pediatric Cardiology, Medical University of Warsaw, Warsaw, Poland
}

Author's address: Danuta Roik, Department of Pediatric Radiology, Medical University of Warsaw, Zwirki i Wigury 63A Str., Warsaw, Poland, e-mail: danroik@gmail.com

\section{Summary}

Computed tomography with its excellent spatial and temporal resolution remains a valuable diagnostic modality in pediatrics. On the other hand an increasing attention has been placed on the radiation risks associated with CT imaging, especially in children.

In recent years, many advances in CT hardware and software, for example, automatic exposure control tools and iterative reconstruction techniques, have allowed for a reduction of applied radiation doses while maintaining image quality.

The purpose of this paper is to present our protocol for chest CT imaging in the youngest age group, together with a pictorial review of congenital pathologies of the chest, and to emphasize factors that optimize postnatal CT imaging in infants.

In our opinion, modern CT imaging with the use of dose reduction techniques and iterative reconstructions allows for a proper visualization of chest pathologies in small children, which has no influence on observer performance. The presented review of low-dose diagnostic images of a wide spectrum of congenital pathologies may serve as an example of routine utilization of the current concepts in pediatric CT optimization.

MeSH Keywords: $\quad$ Congenital Abnormalities • Multidetector Computed Tomography • Neonatology • Pediatrics • Radiation Dosage

PDF file: $\quad$ http://www.polradiol.com/abstract/index/idArt/900615

\section{Background}

Congenital chest pathologies, among which congenital malformations are the most frequent, create a spectrum of developmental abnormalities of the diaphragm, chest wall, foregut, airways, heart and great vessels. The highest incidence is reported for heart and great vessels anomalies [1], but also congenital anomalies of the lungs and airways may cause clinical problems and require diagnostic imaging in small children. Congenital chest lesions may present as large, space-occupying masses that increase the risk of serious complications in fetal life or can cause severe respiratory distress in the neonate; however, currently, most patients referred for an evaluation are asymptomatic neonates and infants with abnormal prenatal ultrasound and magnetic resonance examinations.

Asymptomatic congenital lung lesions may be managed conservatively or by surgical resection. A systematic review and meta-analysis performed to determine the risk associated with elective versus emergency surgery revealed that the risk of asymptomatic cases developing symptoms 
is small [2]. However, elective surgery is associated with a better outcome than emergency surgery and, if considered, should be performed before 10 months of age. Although no prognostic indicators have been so far identified in the literature, a conservative approach may be appropriate for small lesions [2-4].

Diagnostic imaging and management of the vast majority of non-complicated congenital heart diseases can be accomplished by echocardiography alone. Abnormalities beyond the sonographic window, complex 3-dimensional lesions and detailed functional information require additional imaging $[5,6]$.

Computed tomography (CT) offers several advantages over echocardiography and MRI in the evaluation of congenital chest pathologies. Fast acquisition of isotropic datasets improves spatial and temporal resolution of acquired images and reduces the need and/or time of sedation in small babies. It permits high-resolution, multiplanar and threedimensional (3D) reformatting. Image processing techniques have also progressed rapidly and many advances in CT hardware and software, for example, automatic exposure control tools and iterative reconstruction techniques, allow for the reduction of applied radiation doses while maintaining image quality. CT is also less expensive than magnetic resonance (MR) and delivers more information on the lung parenchyma, including the airways [7-9].

We present our protocol for chest CT imaging in the youngest age group, together with a pictorial review of congenital pathologies of the chest examined with the lowest dose indices, and address the most important factors influencing image quality and diagnostic performance.

\section{Case Report}

Our Department of Pediatric Radiology routinely performs chest CT imaging in neonates and infants with congenital pathologies of the chest who are hospitalized in the Department of Pediatric Cardiology and Department of Pediatric Surgery. We reviewed the database of chest CT examinations performed in the youngest group of children (aged 0-5 years), according to body weight protocols (0-10 kg and $10-30 \mathrm{~kg})$. Scans were performed to confirm or exclude suspected congenital pathologies of the lungs, heart and great vessels, chest wall and diaphragm as well as in patients with acquired pulmonary conditions such as infections and broncho-pulmonary dysplasia (BPD). Since 2011, we have been using low-dose chest imaging with a 64-raw scanner, Philips Brilliance. The images were reconstructed with an iterative reconstruction technique, i.e. iDose. We reviewed dose indices, the volume of computed tomography dose index (CTDIvol) and the dose-length product (DLP) obtained or recalculated for a $16-\mathrm{cm}$ phantom to estimate radiation dose and to calculate size-specific dose estimates (SSDE).

Dose indices for the lowest dose scans were as follow: CTDIvol 1.88-3.9 mGy; DLP 29.4-74.9 mGy $\times \mathrm{cm}$; SSDE 2.18-4.71 mGy. The lowest dose indices were obtained in examinations performed in children during pre-operative evaluation of the heart and great vessels with no adverse influence on image quality.

\section{Discussion}

\section{Scanning protocol}

The CT protocol and time of imaging in patients with congenital chest lesions depend on the suspected pathology and the patient's general condition. According to the ALARA-CT concept [10], we focus on avoiding unnecessary radiation during CT in this very young subgroup of patients. Every examination is tailored individually to the patient. We plan CT scanning on the basis of previously performed and analyzed examinations, such as chest X-ray, ultrasound or echo studies and lately also more and more frequently on the basis of prenatally performed MRI.

In most cases, single-phase, enhanced chest angiography is performed, as multiphase scanning should generally be avoided in pediatric patients.

This approach is obvious for patients with congenital cardiac diseases but adds also to definite diagnosis in congenital lung malformations, allowing for better visualization of all tissues in the chest and helping in the differential diagnosis of pulmonary abnormalities.

We prefer not to image neonates in the early post-delivery period to protect their immature kidneys from contrast media and because of the immaturity of other systems, the imbalance in their fluid status and because of the delay in clearance of the lung from the fetal fluid.

Very rarely, in cases of neonates with suspected pulmonary pathology, we perform only unenhanced CT to confirm prenatal findings, especially if they are not visible on chest X-ray and such confirmation is vital for therapeutic decisions.

Iodine, low-osmolar contrast agents (300 Iodine/ml) are warmed to 37 degrees centigrade, to decrease their viscosity and are administered as boluses with a power injector. The amount and flow is individually calculated for every patient to properly cover the scanning time. We use 1-2 ml of contrast medium per kilogram of body weight with a flow rate of $0.8-3.5 \mathrm{ml} / \mathrm{s}$ and a saline flush, calculated individually so as not to exceed the allowed amount of intravenous fluids, especially in very low-weight infants or unstable children.

In most patients, CT is performed with free breathing under short intravenous sedation. Our experience showed that during bolus contrast administration excessive motion artifacts may appear in non-sedated patients, making the scans non-diagnostic, which mostly requires re-scanning.

We use automated bolus triggering, the attenuation within the region of interest (in most cases thoracic aorta) is monitored and the scan is triggered automatically once the enhancement threshold is achieved (130-150 Hounsfield Units [HU]). In children, the inherent delay, the delay between the monitoring series and proper scanning, create important difference between a successful and unsuccessful contrast bolus. The length of the inherent delay depends on the scanner model; in our 64-detector scanner, it is about 4-5 seconds. Adding this 4-5-second delay to the scanning time allows for the calculation of bolus length and time 


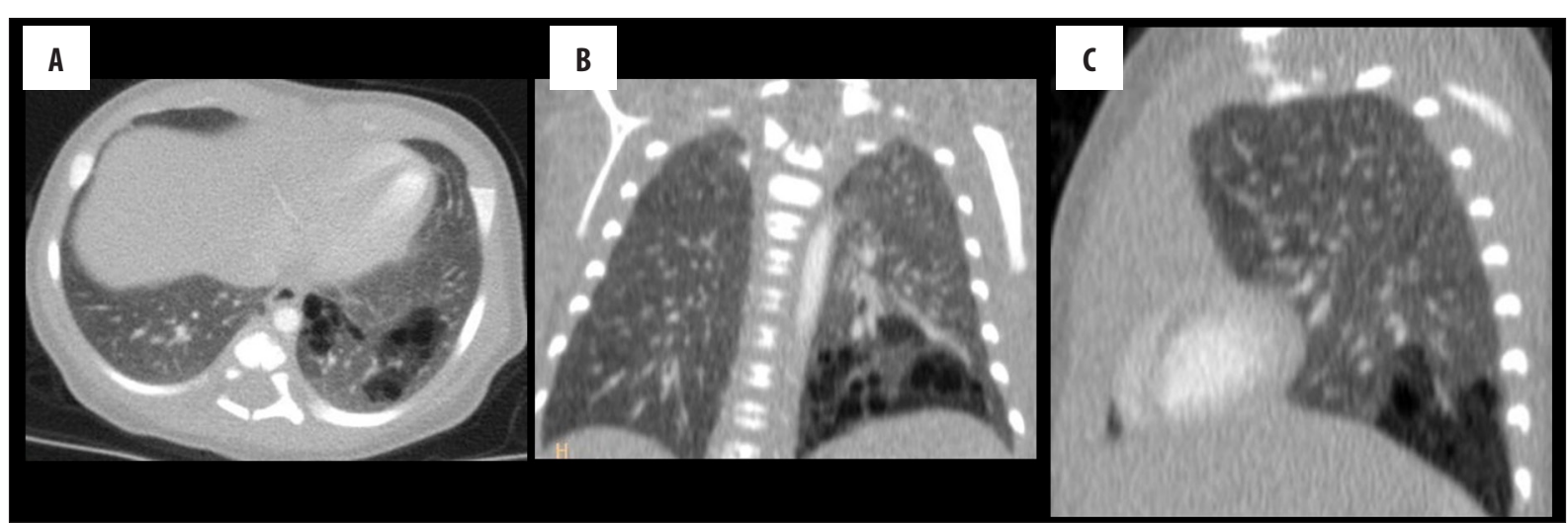

Figure 1. (A-C) Congenital pulmonary airway malformation (CPAM) presenting as air-filled cysts in the left lower lobe in a 13 -day-old girl. $80 \mathrm{kV}$, $50 \mathrm{mAs}$, CTDI 1.88 mGy, DLP $29.4 \mathrm{mGy} \times \mathrm{cm}$, SSDE $2.23 \mathrm{mGy}$.

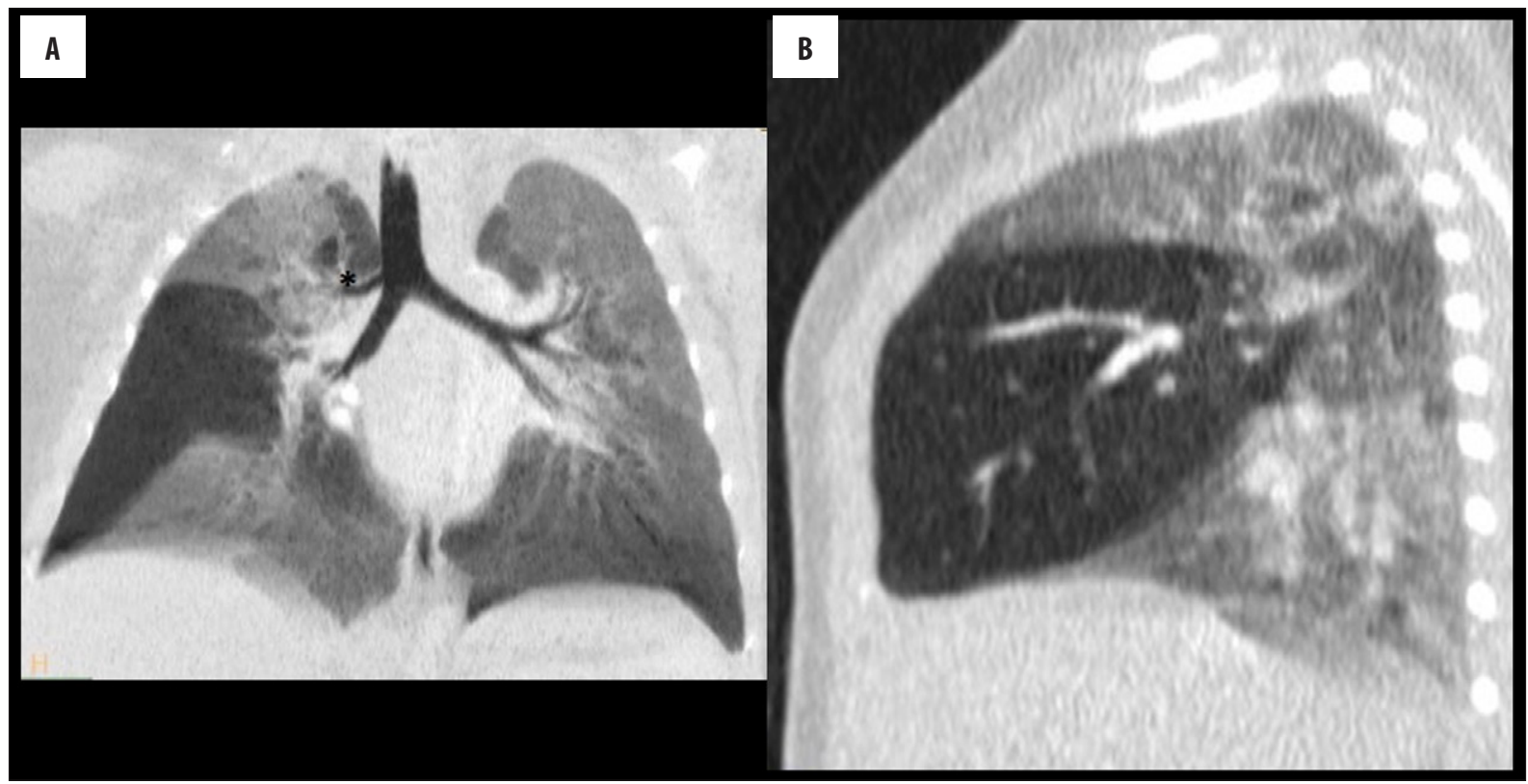

Figure 2. A, B) A seven-month-old girl with congenital heart disease, congenital middle lobe emphysema and tracheal bronchus $\left({ }^{*}\right)$ on the right side. Coronal and sagittal MinIP reconstructions, pulmonary window. 80 kV, 50 mAs, CTDI 1.88 mGy, DLP 34.5 mGy×cm, SSDE 2.18 mGy.

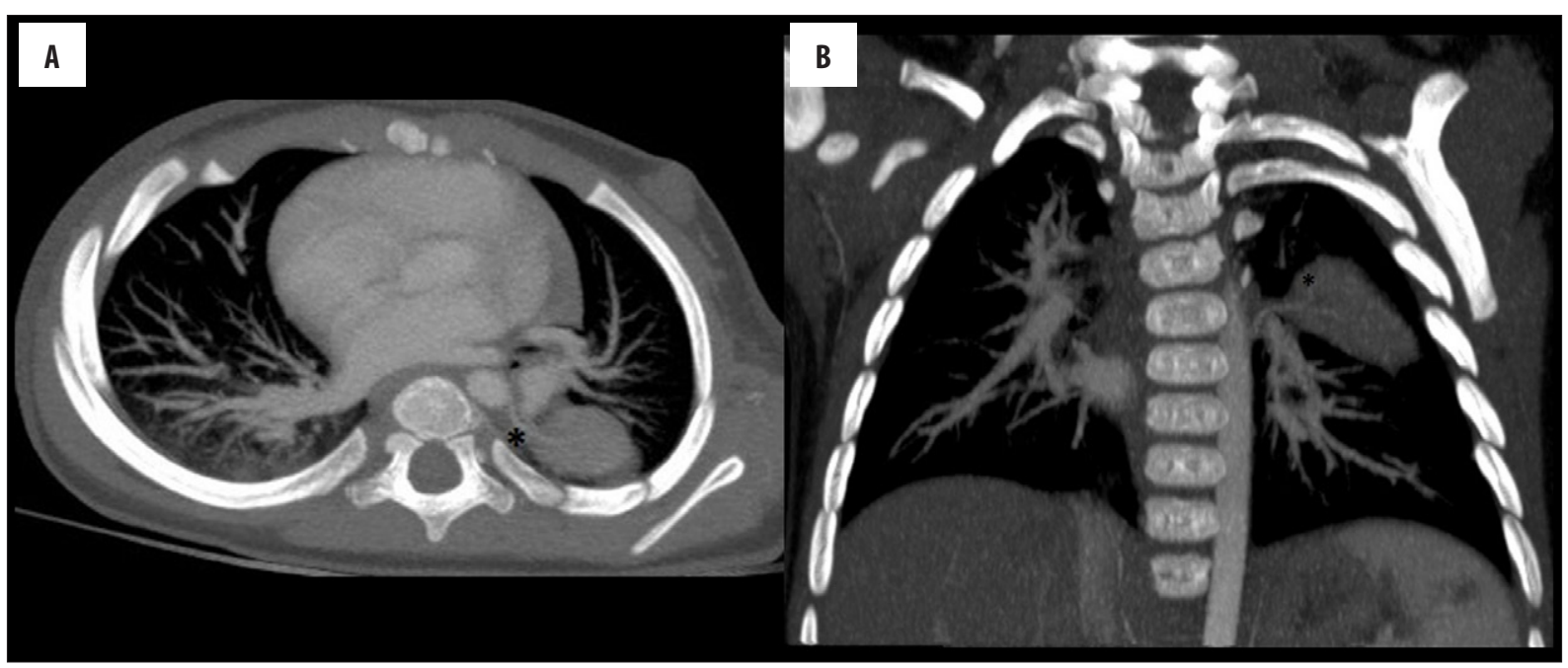

Figure 3. A, B) An eight-month-old girl hospitalized for prenatally detected congenital lung pathology in the upper left lobe. Enhanced CT presents intralobar, pulmonary sequestration with the feeding vessel from the thoracic aorta (*); $100 \mathrm{kV}, 80 \mathrm{mAs}$, CTDI $3.77 \mathrm{mGy}$, DLP 74.3 mGy $\times c m$, SSDE 3.95 mGy. 


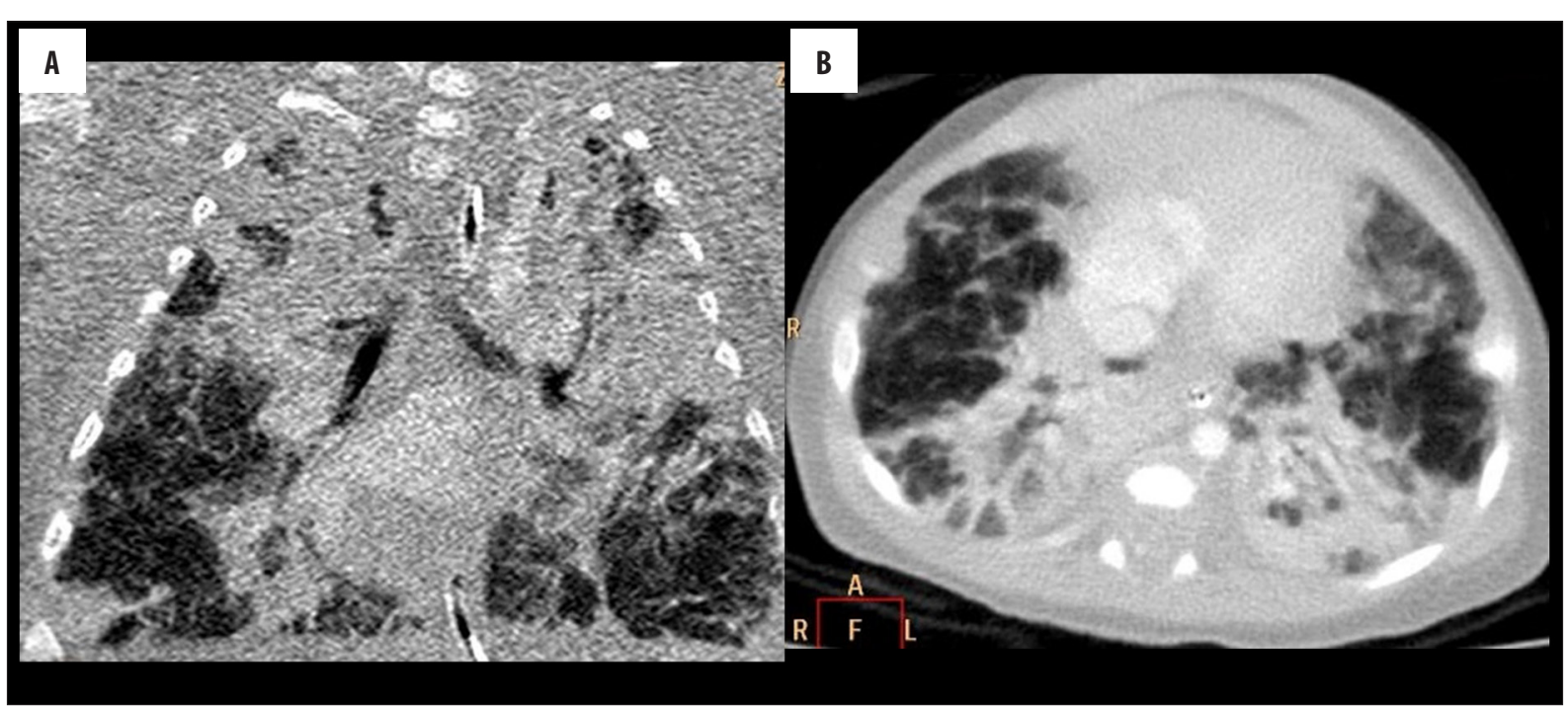

Figure 4. (A, B). Cystic and atelectatic disseminated changes consistent with bronchopulmonary dysplasia in a 3-month-old boy. $80 \mathrm{kV}, 80 \mathrm{mAs}$, CTDI $3.01 \mathrm{mGy}$, DLP $52.1 \mathrm{mGy} \times \mathrm{cm}$, SSDE $3.40 \mathrm{mGy}$.

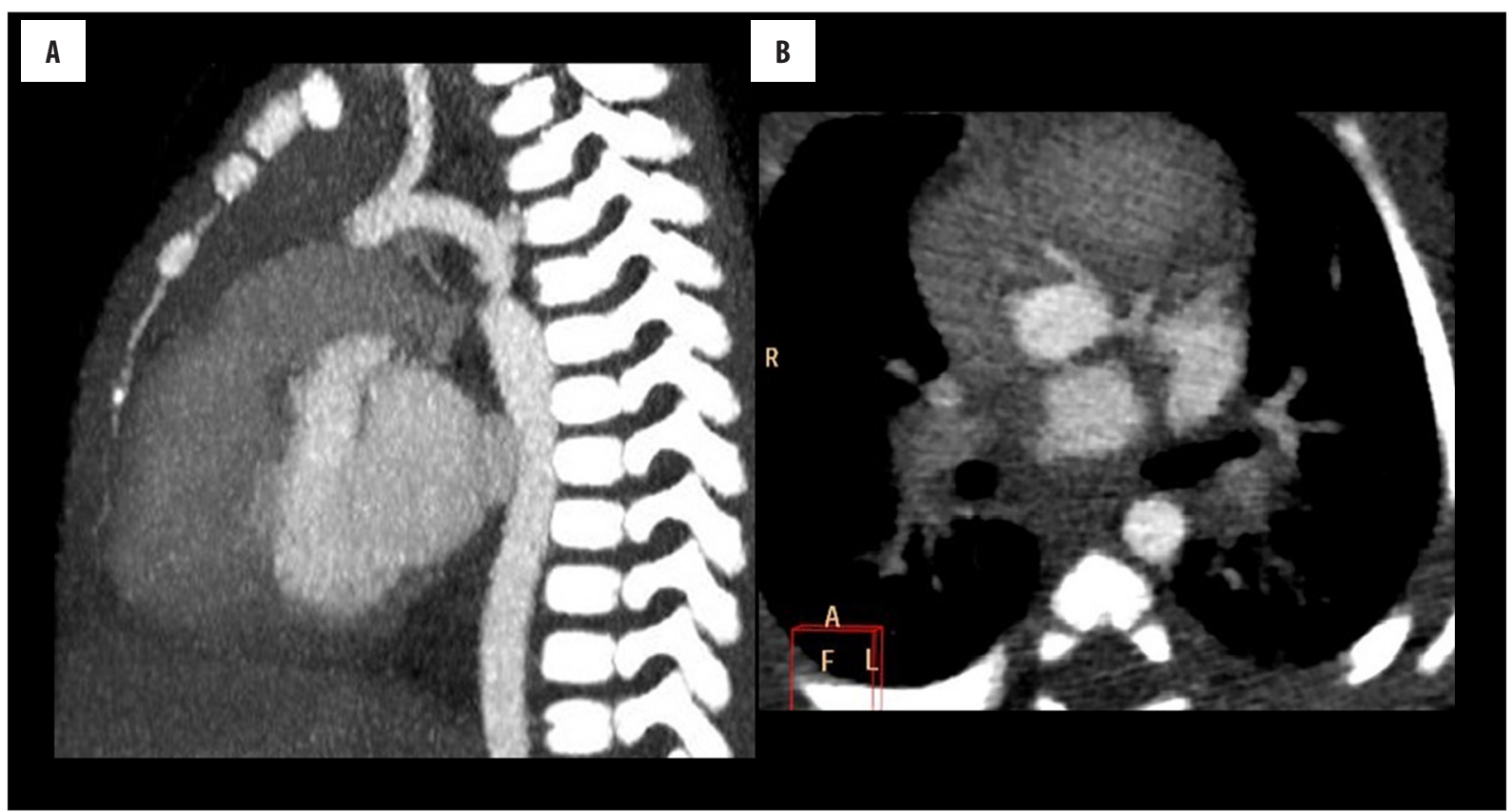

Figure 5. (A-C) A twenty-two-day-old girl with coarctation of the aorta. Image quality allows not only to assess the aortic isthmus stenosis but also the proximal parts of coronary arteries. $80 \mathrm{kV}, 50 \mathrm{mAs}$, CTDI $1.88 \mathrm{mGy}, \mathrm{DLP} 32.5 \mathrm{mGy} \times \mathrm{cm}$, SSDE $2.18 \mathrm{mGy}$.

to acquire the images - this is the traditional approach in which the injection duration is calculated to cover scan time.

\section{Scan parameters optimization}

As the tube voltages during CT angiography should be as low as possible, we scan our patients with $80 \mathrm{kV}$, instead of previously used $100 \mathrm{kV}$. The decrease in tube voltage leads to increased attenuation of the enhanced vascular structures, which is related to the k-edge of iodine $(33.2 \mathrm{keV})$ [11-13]. Routinely used scanning parameters are as follows: tube voltage, $80 \mathrm{kV}$; current, $80-100 \mathrm{~mA}$; if automated tube current modulation is used planned, mAs are of $50-70$. We used collimation of $64 \times 0.625 \mathrm{~mm}$, gantry rotation time of 0.50 seconds and pitch of 0.90 .
Scanning with a lower tube voltage and current results in an increase in the image noise and may compromise image quality and diagnostic performance. This disadvantage was overcome by implementation of the iterative reconstructions in routine clinical practice. Iterative reconstruction techniques more accurately reproduce the process of data acquisition and reduce image noise in comparison to the filtered back projection (FBP) due to an incorporated physical model of CT system. The iterative reconstruction of our scanner, i.e. iDose (Philips Healthcare), works as a hybrid technique that incorporates FBP and an iterative technique with two denoising components $[14,15]$. It is advised to gradually reduce the radiation dose when adopting iterative reconstruction techniques and such a hybrid technique; moreover, blending with FBP appeared to be helpful. As our experience 


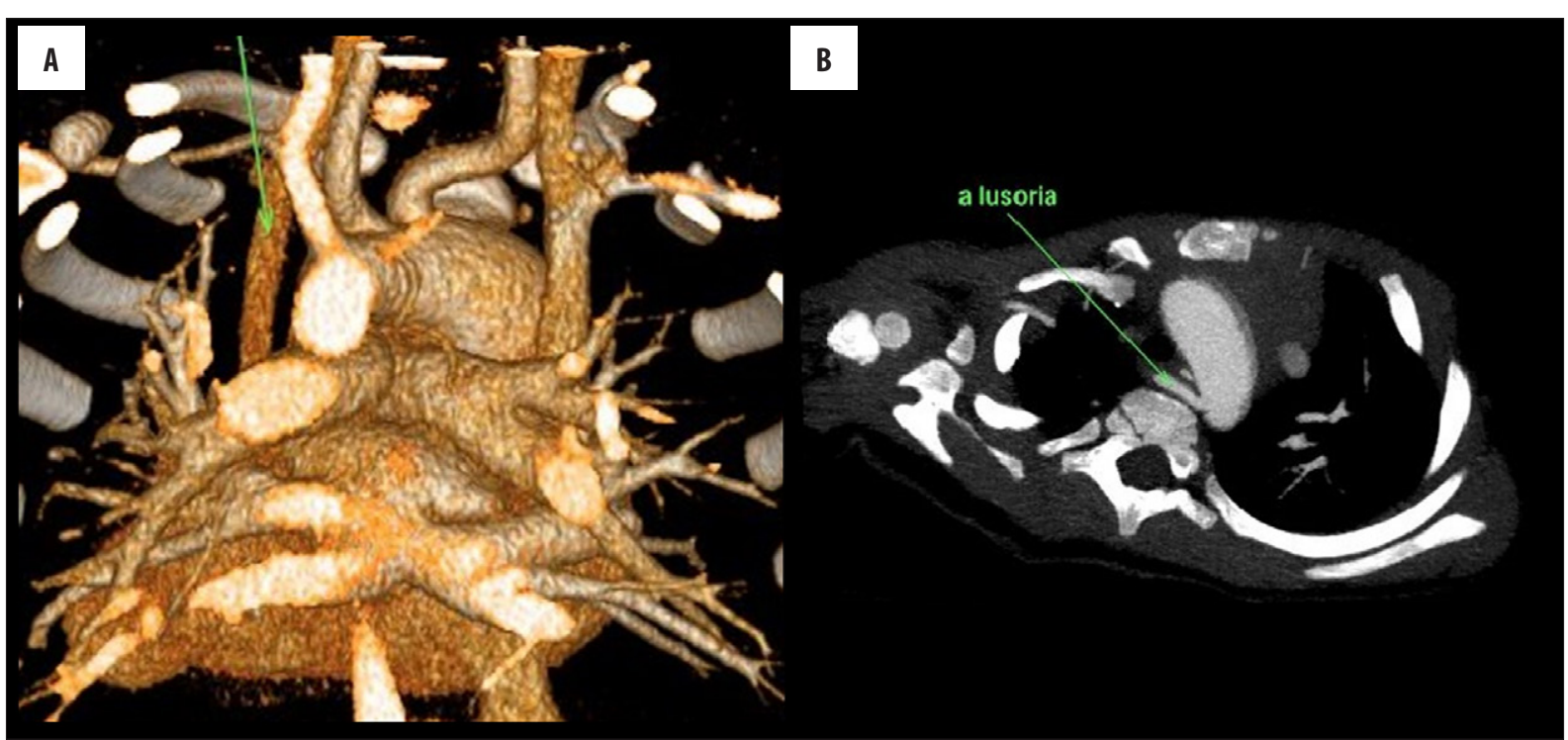

Figure 6. (A, B). An eighteen-month-old girl with aortic stenosis in whom partial anomalous pulmonary venous return (PAPVR) was suspected on echocardiography. Chest CT-angiography excluded PAPVR. An accessory left superior vena cava and arteria lusoria are present. 80 kV, 50 mAs, CTDI 1.8 mGy, DLP 35 mGyXCm, SSDE 2.04 mGy.

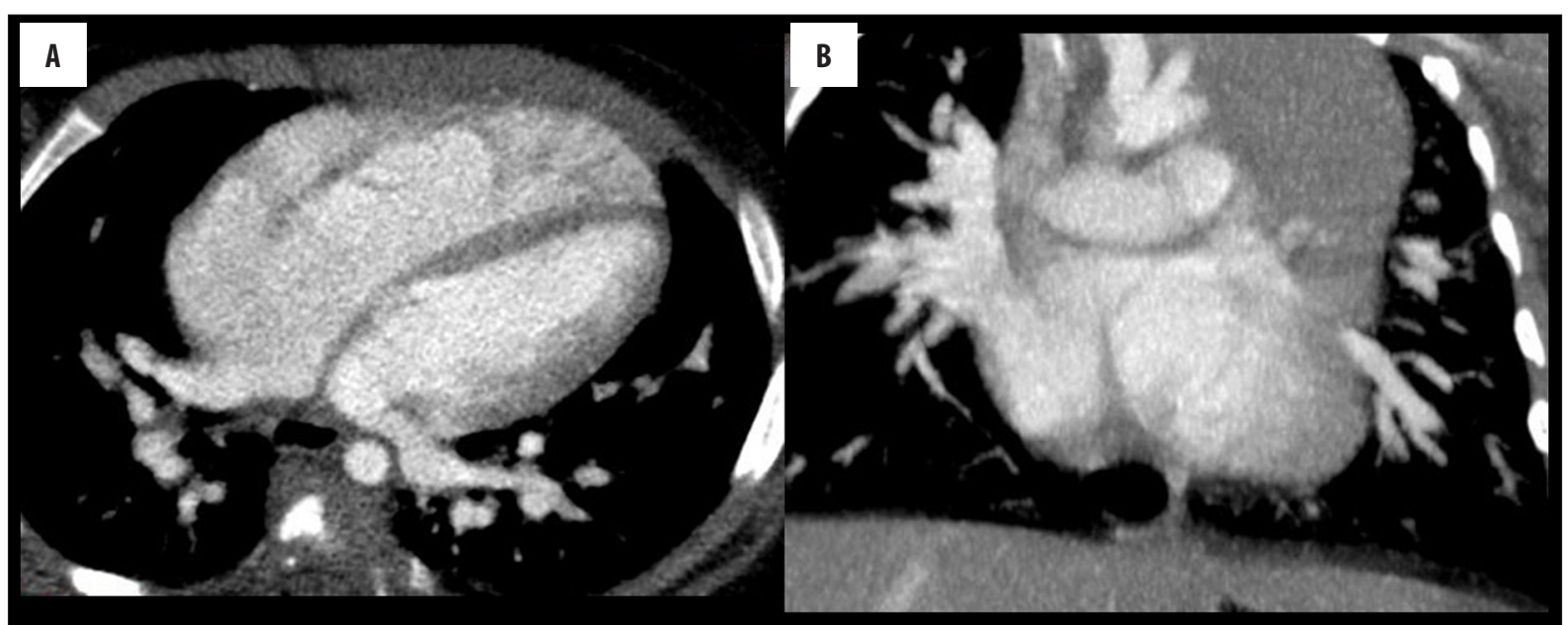

Figure 7. A, B) A three-month-old girl evaluated because of a heart murmur. Chest x-ray revealed increased pulmonary flow. Chest CT showed the right pulmonary veins draining to the right atrium as partial anomalous pulmonary venous return (PAPVR). The left pulmonary veins correctly join the left atrium. 80 kV, 104 mAs, CTDI 3.90 mGy, DLP $62.1 \mathrm{mGy \times cm}$, SSDE $4.71 \mathrm{mGy}$.

with iterative reconstruction has been growing, we have got used to accepting relatively low-dose examination that are noisier than previously achieved with higher scanning parameters reconstructed with the FBP technique. In our opinion, they are sufficient for an adequate evaluation of the airway, lung parenchyma and contrast enhanced vessels in patients with congenital chest pathologies.

Major factors that adversely influence image quality are, in our opinion, suboptimal contrast bolus timing and involuntary movement of patients.

Our experience is concordant with multiple studies showing that iterative reconstruction techniques allow for dose reductions while preserving image quality in the adult and children abdominal or thoracic CT imaging [4,16-19].

\section{Review of pathologies}

A spectrum of pathologies examined with the lowest parameters included:

- congenital pulmonary airway malformation (CPAM) (Figure 1A-1C),

- congenital bronchial abnormalities (Figure2A, 2B),

- pulmonary sequestration (Figure 3A, 3B),

- pulmonary infections; bronchopulmonary dysplasia (BPD) as a complication of respiratory distress syndrome (RDS) (Figure 4A, 4B),

- abnormalities of the aortic arch: right-sided aortic arch, vascular rings, coarctation of aorta (Figures 5A, 5B; 6A, 6B), - partial anomalous pulmonary venous return (PAPVR) (Figure 7A, 7B),

- pulmonary hypoplasia in patients with congenital diaphragmatic hernia (Figure 8A, 8B),

- congenital tumors (Figure 9A-9D) 


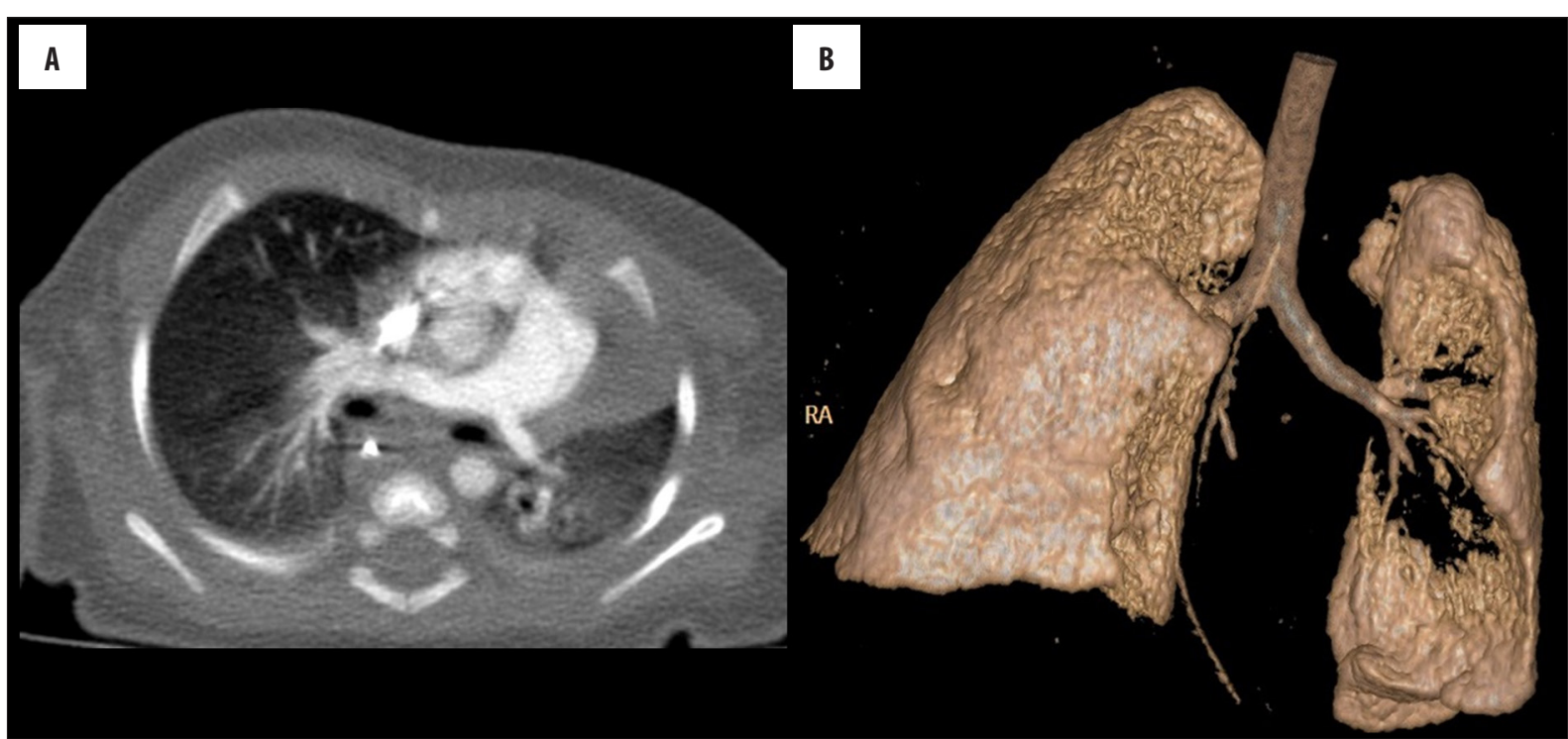

Figure 8. (A, B). A two-month-old girl after surgical treatment of prenatally diagnosed left-sided congenital diaphragmatic hernia. An axial CT scan and volume reconstruction show a hypoplastic, two-lobar left lung and hypoplastic left pulmonary artery. 80 kV, 50 mAs, CTDI $1.88 \mathrm{mGy}$, DLP 30.8 mGy Xcm, SSDE 2.23 mGy.

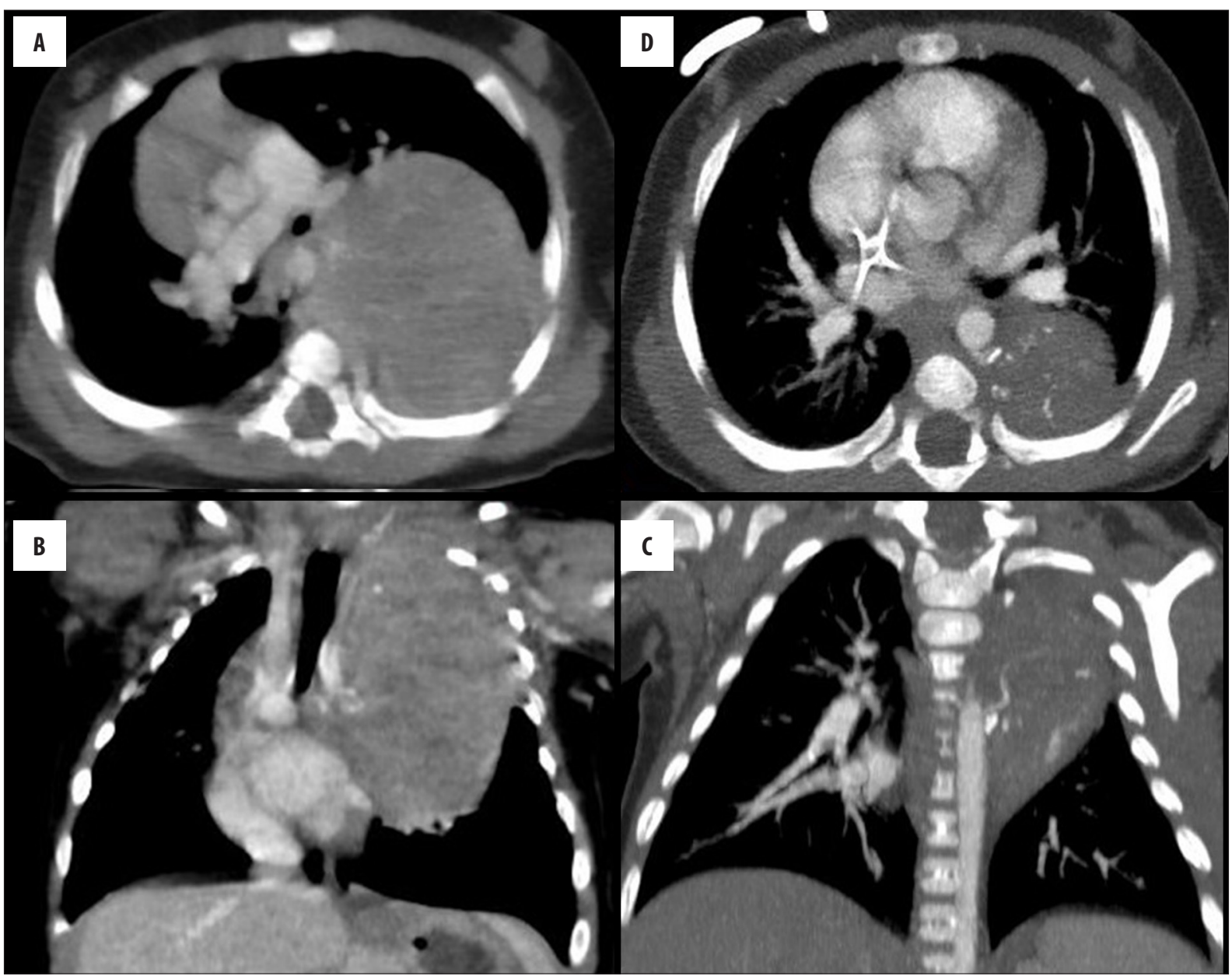

Figure 9. Follow-up studies performed in a 7-month-old girl with a posterior mediastinal tumor (neuroblastoma). Comparison of scanning parameters. An initial CT (A, B) before optimization with the following dose indices: $100 \mathrm{kV}, 100 \mathrm{mAs}$, CTDI 7.72 mGy, DLP 258.1 mGy $\times \mathrm{cm}$, SSDE $8.49 \mathrm{mGy}$; and the follow-up study (C, D) with significantly decreased parameters and no influence on image quality: $80 \mathrm{kV}$, $100 \mathrm{mAs}$, CTDI 3.77 mGy, DLP 74.9 mGy×cm, SSDE 4.07 mGy. 
The anomalies of the aorta, such as the coarctation of the aorta and vascular rings (Figures 5, 6), are the most common indications for CT angiography in infants with congenital heart diseases (CHD). Computed tomography is considered as the optimal modality as it allows for a simultaneous assessment of vessels and the tracheobronchial tree (Figure 2). In current algorithms, chest CT angiography has already completely replaced catheterization, not only in the above-mentioned cases, but also as non-invasive imaging that can establish the anatomic details required for surgical interventions for most CHDs [6].

Chest CT angiography is also well-established in the evaluation of pulmonary artery and veins anomalies (Figures 7, 8), and in differential diagnosis between congenital pulmonary lesions such as sequestration with abnormal arterial supply and congenital pulmonary airway malformation (CPAM) (Figures 1, 3).

Imaging of pulmonary pathologies in small children can be very challenging due to the small size of the lesions and supplying vessels or very discreet differences in lung aeration (Figures 2,4). Those problems can be overcome by choosing appropriate time for scanning, scan parameters and by the use of novel reconstruction techniques. There are studies that confirm superior depiction of small vessels in pediatric patients undergoing contrast-enhanced CT of the chest and abdomen reconstructed with model-based iterative reconstructions $[16,20]$.

The need for CT optimization in children undergoing repeated scans for cancer staging and follow-up is obvious. These patients benefit most from the use of low-dose CT imaging together with iterative reconstruction techniques for noise reduction (Figure 9).

\section{Conclusions}

Chest pathologies can be properly visualized in small children using iterative CT reconstruction techniques with an appropriate quality of scans at a reduced dose with no influence on observer performance.

In our opinion, observer performance is mostly influenced in cases of inappropriate contrast bolus timing and when severe motion artifacts appear, rather than by more noisy, reduced dose CT scans.

Our protocol for chest CT in the youngest group of patients and a review of low-dose diagnostic images of a wide spectrum of chest congenital pathologies may serve as an example of routine utilization of the current concepts in pediatric CT optimization.

\section{References:}

1. Dolk H, Loane M, Garne E: The prevalence of congenital anomalies in Europe. Adv Exp Med Biol, 2010; 686: 349-64

2. Stanton M, Njere I, Ade-Ajayi $\mathrm{N}$ et al: Systematic review and metaanalysis of the postnatal management of congenital cystic lung lesions. J Pediatr Surg, 2009; 44: 1027-33

3. Newman B: Congenital bronchopulmonary foregut malformations: Concepts and controversies. Pediatr Radiol, 2006; 36: 773-91

4. Epelman M, Kreiger PA, Servaes S et al: Current imaging of prenatally diagnosed congenital lung lesions. Semin Ultrasound CT MR, 2010; 31(2): 141-57

5. Chan FP, Hanneman K: Computed tomography and magnetic resonance imaging in neonates with congenital cardiovascular disease. Semin Ultrasound CT MR, 2015; 36(2): 146-60

6. Han BK, Rigsby CK, Hlavacek A et al: Computed tomography imaging in patients with congenital heart disease part I: Rationale and utility. an expert consensus document of the society of cardiovascular computed tomography (SCCT): Endorsed by the Society of Pediatric Radiology (SPR) and the North American Society of Cardiac Imaging (NASCI). J Cardiovasc Comput Tomogr, 2015; 9(6): 475-92

7. Frush DP, Herlong JR: Pediatric thoracic CT angiography. Pediatr Radiol, 2005; 35: 11-25

8. Lee EY, Kritsaneepaiboon S, Zurakowski D et al: Beyond the pulmonary arteries: Alternative diagnoses in children with MDCT pulmonary angiography negative for pulmonary embolism. Am J Roentgenol, 2009; 193: 888-94

9. Pacharn P, Kline-Fath B, Calvo-Garcia M et al: Congenital lung lesions: Prenatal MRI and postnatal findings. Pediatr Radiol, 2013; 43: $1136-43$

10. Pediatr Radiol (2011) 41 (Suppl 2): ALARA-CT.
11. Kalva SP, Sahani DV, Hahn PF: Using the K-edge to improve contrast conspicuity and to lower radiation dose with a 16-MDCT: A phantom and human study. J Comput Assist Tomogr, 2006; 30: 391-97

12. Herzog C, Mulvihill DM, Nguyen SA: Pediatric cardiovascular CT angiography: Radiation dose reduction using automatic anatomic tube current modulation. Am J Roentgenol, 2008; 190: 1232-40

13. Singh S, Kalra MK, Moore MA: Dose reduction and compliance with pediatric CT protocols adapted to patient size, clinical indication, and number of prior studies. Radiology, 2009; 252: 200-8

14. Seibert JA: Iterative reconstruction: How it works, how to apply it. Pediatr Radiol, 2014; 44(Suppl. 3): 431-39

15. Khawaja RD, Singh S, Otrakji A et al: Dose reduction in pediatric abdominal CT: Use of iterative reconstruction techniques across different CT platforms. Pediatr Radiol, 2015; 45(7): 1046-55

16. Haggerty JE, Smith EA, Kunisaki SM, Dillman JR: CT imaging of congenital lung lesions: Effect of iterative reconstruction on diagnostic performance and radiation dose. Pediatr Radiol, 2015; 45(7): 989-97

17. Singh S, Kalra MK, Hsieh J: Abdominal CT: Comparison of adaptive statistical iterative and filtered back projection reconstruction techniques. Radiology, 2010; 257: 373-83

18. Kaza RK, Platt JF, Goodsitt MM: Emerging techniques for dose optimization in abdominal CT. Radiographics, 2014; 34: 4-17

19. Gonzalez-Guindalini FD, Ferreira Botelho MP, Töre HG: MDCT of chest, abdomen, and pelvis using attenuation-based automated tube voltage selection in combination with iterative reconstruction: An intrapatient study of radiation dose and image quality. Am J Roentgenol, 2013; 201(5): 1075-82

20. Koc G, Courtier JL, Phelps A et al: Computed tomography depiction of small pediatric vessels with model-based iterative reconstruction. Pediatr Radiol, 2014; 44: 787-94 